\title{
RELATIONSHIP BETWEEN EQUITABLE TOTAL COLORING AND RANGE COLORING IN SOME REGULAR GRAPHS
}

\author{
Abel Rodolfo García Lozano ${ }^{1 *}$, Angelo Santos Siqueira ${ }^{2}$, \\ Clícia Valladares Peixoto Friedmann ${ }^{3}$ and Samuel Jurkiewicz Jurkiewicz ${ }^{4}$
}

Received February 18, 2015 / Accepted March 21, 2016

\begin{abstract}
This work aims to study the equitable total coloring into subfamilies of regular graphs. For this purpose, we use some relationships between equitable total coloring and range (vertex) coloring in some regular graphs. The concept of range coloring of order $k$ was first presented by Lozano et al. (2009). In this paper, we shows that if a regular graph $G$ admits an equitable range coloring $c$ of order $\Delta$ with $(\Delta+1)$ colors then there is an equitable total coloring of $G$ - with the same set of colors - that extends $c$. We also show that there are infinite graphs satisfying this theorem. Such graphs are called Harmonics. We generate Harmonic Graphs which are Cartesian products of cycles and their complements. These graphs are regular and they admit an equitable total coloring under the above conditions.
\end{abstract}

Keywords: equitable total coloring, range coloring, regular graphs.

\section{BASIC CONCEPTS AND NOTATIONS}

In this section, we present some basic definitions and notations used in this paper, and that can be found in Bondy \& Murty (1976), Diestel (1997) or Yap (1986). Let $G(V, E)$ or $G$ denote a graph with vertex set $V$ and edge set $E$. All graphs considered in this text are finite and simple.

\subsection{Basic concepts}

A graph $G(V, E)$ is $k$-regular if all its vertices have the same degree $k$. A complete graph on $n$ vertices is a $(n-1)$-regular graph; and it is denoted $K_{n}$. Let $M$ be a matching in a graph $G$; a vertex $v$ of $G$ is said $M$-saturated by $G$ if some edge of $M$ is incident to $v$; otherwise $v$ is $M$-unsaturated. A matching that saturates all vertices of $G$ is called a perfect matching.

\footnotetext{
*Corresponding author.

${ }^{1}$ Departamento de Matemática da FFP/UERJ. Programa de Pós-Graduação em Ensino das Ciências da ECELAH/UNIGRANRIO, Rio de Janeiro, RJ, Brasil. E-mail: arglozano@ terra.com.br

${ }^{2}$ Programa de Pós-Graduação em Humanidades, Culturas e Artes da ECELAH/UNIGRANRIO, Rio de Janeiro, RJ, Brasil. E-mail: asiqueira@unigranrio.edu.br.

${ }^{3}$ Departamento de Matemática da FFP/UERJ, Rio de Janeiro, RJ, Brasil. E-mail: cliciavp@ @erra.com.br

${ }^{4}$ Programa de Engenharia da Produção da COPPE/UFRJ, Rio de Janeiro, RJ, Brasil. E-mail: jurki@ pep.ufrj.br
} 


\subsection{Notations}

Let $G$ be a graph. We will adopt the following notations:

$d_{G}(v)$ or $d(v)$ denotes the degree of a vertex $v \in G$.

$\delta(G)$ or $\delta$ denotes the minimum degree of $G$.

$\Delta(G)$ or $\Delta$ denotes the maximum degree of $G$.

$N_{G}(v)$ or $N(v)$ denotes the open neighborhood of a vertex $v \in G$.

In the next two following sections, we will present other definitions that are relevant to understand this paper.

\section{CARTESIAN PRODUCT OF GRAPHS}

The Cartesian product $G \square H$ of two graphs $G$ and $H$ with vertex sets $V(G)$ and $V(H)$ is the graph such that its vertex set is $V(G) \square H(G)$. Any two vertices $(g, h)$ and $\left(g^{\prime}, h^{\prime}\right)$ of the $G \square H$ are adjacent whenever $g=g^{\prime}$ and $h$ is adjacent to $h^{\prime}$ in $H$ or $h=h^{\prime}$ and $g$ is adjacent with $g^{\prime}$ in $G$. This definition can be found in Kemnitz \& Marangio (2003), Seoud et al. (1997) or Zmazek \& Zerovnik (2002).

Figure 1 illustrates the Cartesian product $P_{3} \square C_{4}$ of the Path 3 and the Cycle 4.
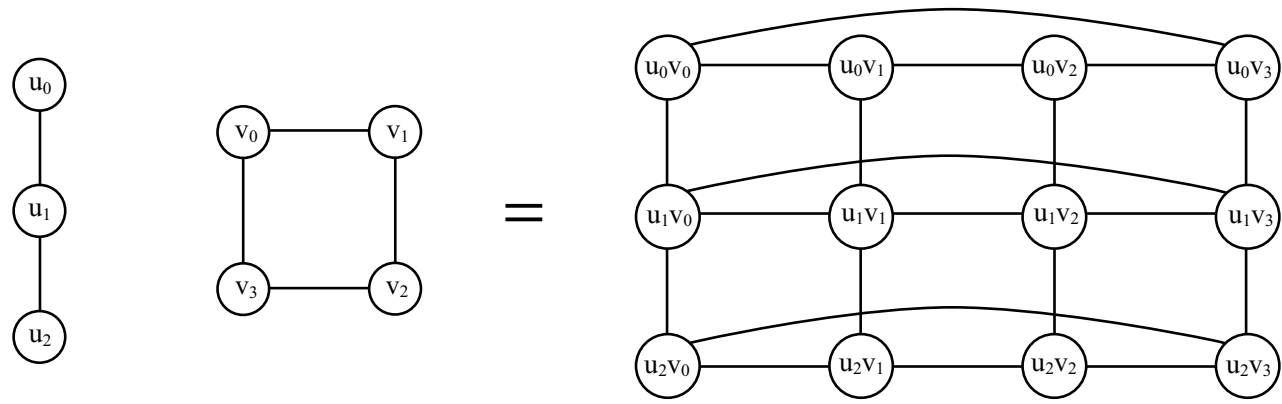

Figure 1 - Cartesian product $P_{3} \square C_{4}$ of a path of 3 vertices and a cycle of 4 vertices.

\section{VERTEX COLORING, TOTAL COLORING AND EQUITABLE TOTAL COLORING}

Let us consider a graph $G(V, E)$, a set $S \subset(V \cup E)$ and a finite set of colors $C$.

A coloring $c: S \rightarrow C$ of $G$ is proper if no two adjacent elements of $S$ share a common color of $C$. If $S=V$ then the proper coloring is a vertex coloring. If $S=E$ or $S=V \cup E$ then we have, respectively, an edge coloring and a total coloring. In this paper, all colorings are proper. A coloring $c$ is equitable if for every $c_{1}, c_{2} \in C,\left|a\left(c_{1}\right)-a\left(c_{2}\right)\right| \leq 1$, where $a\left(c_{1}\right)=\left|c^{-1}\left(c_{1}\right)\right|$ and $a\left(c_{2}\right)=\left|c^{-1}\left(c_{2}\right)\right|$. 
The smallest number $k$ such that $G$ has a vertex coloring with $k$ colors is the chromatic number of $G$, denoted by $\chi(G)$ or $\chi$. We can extend this definition to edge chromatic number of $G$ and to total chromatic number of $G$. They are denoted respectively by $\chi^{\prime}(G)$ or $\chi^{\prime}$ and $\chi^{\prime \prime}(G)$ or $\chi^{\prime \prime}$.

The equitable chromatic number of $G$, denoted $\chi_{e}(G)$ or $\chi_{e}$ is the smallest number $k$ such that $G$ has a vertex equitable coloring with $k$ colors. Also, we can extend this definition to edge equitable chromatic number and to total equitable chromatic number of $G$. They are denoted respectively by $\chi_{e}^{\prime}(G)$ or $\chi_{e}^{\prime}$ and $\chi_{e}^{\prime \prime}(G)$ or $\chi_{e}^{\prime \prime}$.

These concepts can be studied with more details in Chen et al. (1994a), Chen et al. (1994b), Chen et al. (1996), Chunling et al. (2009), Hilton \& Werra (1994), Lozano (2005), Lozano et al. (2008), Siqueira (2011), Wang \& Zhang (2000) and Yap (1996).

Figure 2 illustrates a 4-equitable total coloring with the colors $c_{1}, c_{2}, c_{3}$ and $c_{4}$. Observe that $a\left(c_{1}\right)=a\left(c_{2}\right)=a\left(c_{4}\right)=4$ and $a\left(c_{3}\right)=5$. In this case, $\chi_{e}^{\prime \prime}(G)=4$.

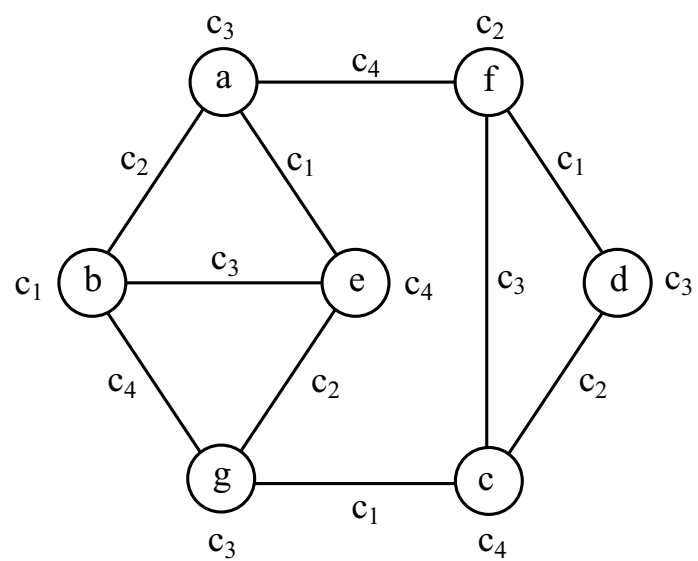

Figure 2 - A 4-equitable total coloring.

Clearly, for any graph $G, \chi^{\prime \prime} \geq \Delta+1$ and $\chi^{\prime \prime} \geq \chi_{e}^{\prime \prime}$. Then the equitable total chromatic number has the same lower bound as the total chromatic number of $G$. The Conjecture of Wang for equitable total coloring, in Wang (2002), says that $\chi_{e}^{\prime \prime} \leq \Delta+2$, i.e. thus, it is desirable to find a total equitable coloring that meets the conjecture.

Let $G(V, E)$ be a graph; $C$ and $C^{\prime}$ finite sets of colors $\left(C \subset C^{\prime}\right) ; c: V \rightarrow C$ a vertex coloring of $G$; and $c^{\prime}:(V \cup E) \rightarrow C^{\prime}$ a total coloring of $G$. We say that the coloring $c^{\prime}$ extends $c$ or $c^{\prime}$ is an extension of $c$ if, for all $v \in V, c^{\prime}(v)=c(v)$.

\section{RANGE COLORING}

The concept of range coloring of order $k$ was first presented by Lozano et al. (2009). After that, Friedmann et al. (2011) proved a theorem that provides a technique to show that some families of graphs satisfy the Total Coloring Conjecture and it was used to color totally the Cactus-Graphs. 
In this paper, the same technique helps us to get a total equitable coloring for a family of regular graphs, which are Cartesian products of Cycles and their complements. Before that, we define range coloring of order $k$ and range chromatic number of order $k$ of a graph.

Let $G=(V, E)$ be a graph and $k \in \mathbb{N}$. A vertex coloring $c: V \rightarrow C$ of $G$ is called a range vertex coloring of order $k$ of $G$, if for all $v \in V$ such that $d(v)=k,|c(N(v))|=d(v)$; otherwise $|c(N(v))| \geq k$, where $|c(N(v))|$ is the number of colors appearing in the open neighborhood of $v$.

Observe that, in a range coloring of order $k$, vertices of degree less than or equal to the required range must have all their neighbors colored with distinct colors, and vertices of degree greater than $k$ need at least $k$ colors in their neighborhood. Moreover, if $k=1$ we have the usual vertex coloring, $k=\Delta$ is equivalent to a 2-distance coloring and it is an upper bound for the order of a range coloring.

Figure 3 below illustrates the coloring of a cycle of 4 vertices (C4). In 3(A), a usual vertex coloring of the cycle is shown while in $3(\mathrm{~B})$ the coloring of the vertices is of range 2.

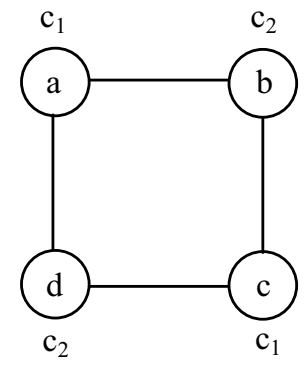

(A)

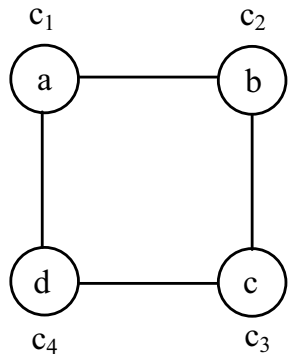

(B)

Figure 3 - Coloring of a C4.

A graph $G$ is $t$-range colorable of order $k$ if there is a range coloring $c$ of order $k$ that uses $t$ colors. The range chromatic number of order $k$ of $G$, denoted $\chi_{f}^{k}(G)$, is the smallest value of $t$ for which $G$ is $t$-range colorable of order $k$.

Figure 4 shows range colorings of different orders for an octahedron. In 4(A), the range coloring is of order 2 and $\chi_{f}^{2}(G)=\chi(G)=3$. Figure 4(B) shows a range coloring of order 3 and $\chi_{f}^{3}(G)=5$. Finally, in Figure $4(\mathrm{C})$, the range coloring is of order 4 and $\chi_{f}^{4}(G)=6$.

\section{RELATIONSHIP BETWEEN THE EQUITABLE TOTAL COLORING AND THE RANGE VERTEX COLORING IN SOME REGULAR GRAPHS}

The main objective of this section is to show the relationship between the equitable total coloring and the range vertex coloring in some regular graphs. For this, we present five theorems which describe this relationship. We just enunciate the Theorem 1, which was proved by Friedmann et al. (2011). The second one shows that, if a range coloring of a graph $G$ is of order $\Delta$, using 

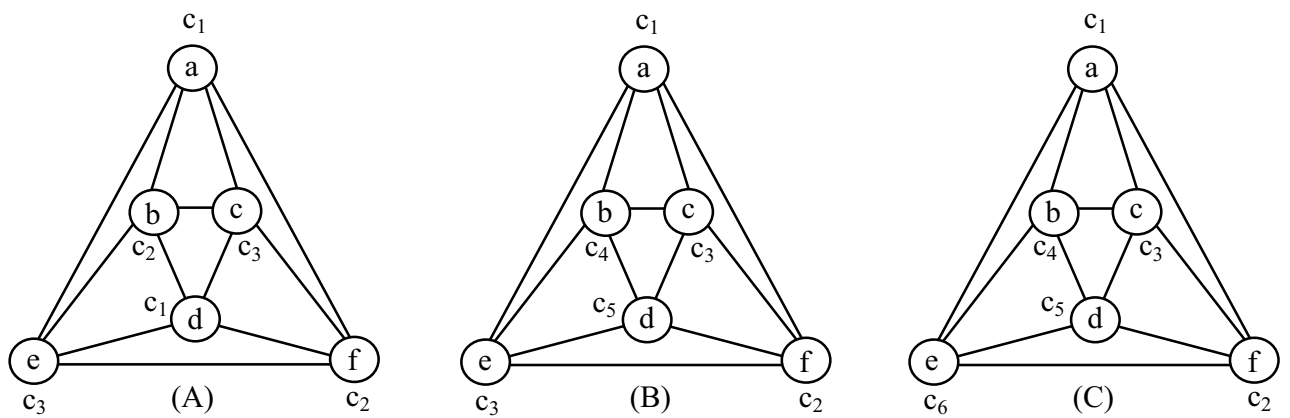

Figure 4 - Range coloring of order 2, 3 and 4, respectively.

$(\Delta+1)$ colors, then there is a total coloring of $G$ satisfying the Vizing-Behzad Conjecture. It is a particular case of the Theorem 1. The third theorem proves that a range coloring of order $\Delta$, using $\Delta+1$ colors, of a regular graph is equitable. The fourth one shows that if a range coloring of a regular graph $G$ is of order $\Delta$, using $\Delta+1$ colors, then the total coloring of the graph satisfies the Wang's Conjecture and it is equitable.

We suggest the name Harmonic Graph for any regular graph that has a range coloring of order $\Delta$, using $\Delta+1$ colors. At first, one might think that there are few Harmonic Graphs, but Lozano et al. (2013) showed ways to obtain some of these graphs.

The last theorem of this section proves that the Cartesian product of Cycles and their complements are Harmonic Graphs. As a consequence of Theorem 4, the total coloring of these graphs is equitable and they meet the Wang's Conjecture.

Theorem 1 (7). Let $G(V, E)$ be a graph with maximum degree $\Delta$ and $c: V \rightarrow C=\{1,2, \ldots, t\}$ a range coloring of order $\Delta$ of $G$. Then, there is a total coloring of $G$ with at most $(t+1)$ colors.

Theorem 2. Let $G(V, E)$ be a graph with maximum degree $\Delta$ and $c: V \rightarrow C=\{1,2, \ldots, \Delta+$ 1 ) a range coloring of order $\Delta$ of $G$. Then, there is a total coloring of $G$ satisfying the Total Coloring Conjecture.

Proof. Let $H_{\Delta+1}\left(V^{\prime}, E^{\prime}\right)$ be the complete graph of order $(\Delta+1)$. It is known that $H_{\Delta+1}$ can be totally colored with $(\Delta+1)$ or $(\Delta+2)$ colors and its vertex coloring is a range coloring of order $(\Delta+1)$. Let $c^{\prime}: V \rightarrow C^{\prime}=\{1,2, \ldots, \Delta+2\}$ be a total coloring of $H_{\Delta+1}$. Without loss of generality, we identify each vertex of $H_{\Delta+1}$ with the color associated to it by the total coloring $c^{\prime}$ such that the color of each vertex of $H_{\Delta+1}$ belongs to the set $\{1,2,3, \ldots, \Delta+1\}$.

We use the total coloring $c^{\prime}$ of $H_{\Delta+1}$ to extend the range coloring $c$ of order $\Delta$ of the vertices of graph $G$ to a total coloring of $G$. First, we define the mapping $f: V \rightarrow V^{\prime}: f(u)=i$ where $i$ is the vertex of $H_{\Delta+1}$ such that $c(u)=c^{\prime}(i)=i$. Now, for each edge $u v \in E$, we define $c(u v)=c^{\prime}(f(u) f(v))$. The coloring defined is proper, since if two edges $u v$ and $u v^{\prime}$ are incident in $u$ then $c(v) \neq c\left(v^{\prime}\right)$, hence $f(v) \neq f\left(v^{\prime}\right)$ and as $c^{\prime}(f(u) f(v)) \neq c^{\prime}\left(f(u) f\left(v^{\prime}\right)\right)$, 
then $c(u v) \neq c\left(u v^{\prime}\right)$. On the other hand, if $u v \in E$ is an edge incident on vertex $v \in V$, then $c^{\prime}(f(v)) \neq c^{\prime}(f(u) f(v))$, therefore $c(v) \neq c(u v)$.

The total coloring obtained by the technique used in the proof of Theorem 2 is called the natural extension of a range coloring of order $\Delta$ of $G$.

Theorem 3. Let $G(V, E)$ be a regular graph, and $c: V \rightarrow C=\{1,2, \ldots, \Delta+1\}$ a range coloring of order $\Delta$ of $G$. Then:

a) $\Delta+1$ divides $|V|$;

b) Each color $i \in C$, is used exactly $\frac{|V|}{\Delta+1}$ times.

Proof. For each color $i \in C, V_{i}$ is the set of vertices with color $i$ by $c$. In order to proceed the demonstration, the following facts have to be observed:

Fact 1. For all $i \in C, V_{i}$ is an independent set of $V$. As $d(v)=\Delta$, (remember that $G$ is a regular graph), all colors of $C-\{c(v)\}$ are used in the neighborhood of vertex $v(N(v))$, i.e. , if $c(v) \neq i$, there is a vertex $u \in V_{i} \cap N(v)$.

Fact 2. For every $i \in C$, if two vertices $u, v \in V_{i}$ then $N(u) \cap N(v)=\varnothing$. As a consequence of a range coloring of order $\Delta$, all neighbors of any vertex $w$ of $G$ must have different colors.

Now, given $i, j \in C$, with $i \neq j$, let $f: V_{i} \rightarrow V_{j}$ be a mapping constructed as follows: $f(u)=v$, if and only if $v \in V_{j} \cap N(u)$.

According to the Facts 1 and 2, the mapping $f$ is a bijection. Then $\left|V_{i}\right|=\left|V_{j}\right|$ and the assertions a) and b) of theorem are direct consequences of this result.

Observe that the above range coloring of order $\Delta$ of $G$ is equitable. Now, we prove that the natural extension of the range coloring of order $\Delta$ of $G$ is an equitable total coloring of $G$.

Theorem 4. Let $G(V, E)$ be a regular graph, and $c: V \rightarrow C=\{1,2, \ldots, \Delta+1\}$ a range coloring of order $\Delta$ of $G$. Then the natural extension of $c$ is an equitable total coloring.

Proof. We name $c^{\prime}$ a natural extension of the coloring $c$ of the graph $G$ and $C^{\prime}$ the set $\{1,2, \ldots$, $\Delta+2$ \}. For each color $i \in C^{\prime}, E_{i}$ is the set of edges with the color $i$ by $c^{\prime}$. This proof is divided into two cases:

Case 1. The color $(\Delta+2)$ was not used in the total coloring, then $\Delta$ is even. Some facts are taken into consideration:

Fact 3. Given $i \in C$ and $v \in V ; c(v) \neq i$ then there is an edge $e \in E_{i}$, such that $v$ is an end of $e$. As $d(v)=\Delta$, all colors $C-\{c(v)\}$ are used on edges incident on $v$ but as $c(v) \neq i$ then there is an edge incident on $v$ at color $i$. 
Fact 4. Given $i \in C$, let be $V^{\prime}=V-V_{i}$ and let $G^{\prime}\left(V^{\prime}, E^{\prime}\right)$ be the subgraph of $G$ induced by $V^{\prime}$ and consider $c^{\prime \prime}$ the restriction of coloring $c^{\prime}$ to $G^{\prime}$ then $E_{i}$ is a perfect matching of $G^{\prime}$. Remember that a perfect matching is the one that covers all vertices of $G^{\prime}$. The edge set $E_{i}$ is a matching because it is a proper coloring and it is perfect according to Fact 3.

As a consequence of Facts 3 and 4, each color $i \in C$ is used exactly $\frac{\left|V-V_{i}\right|}{2}$ times in the edges which it proves that $\left|V-V_{i}\right|$ is even.

Case 2. The color $(\Delta+2)$ was used, then $\Delta$ is odd.

Fact 5. For every vertex $v \in V$, there is an edge $e$ incident to $v$ associated to color $(\Delta+2)$. To prove it, just remember that for each vertex $u \in V, d_{G}(u)=d_{H_{\Delta+1}}(f(u))=\Delta$, where $H_{\Delta+1}$ is the complete graph of order $(\Delta+1)$. Therefore, the set of colors used to color the edges incident on $u$ in graph $G$ is exactly the same set used for coloring the edges incident on $f(u)$ on graph $H_{\Delta+1}$; and that color $(\Delta+2)$ is used on some edge incident on $f(u)$. Thus, the color $(\Delta+2)$ is used $\frac{|V|}{2}$ times, since $|V|$ is even, because $G$ is regular of odd degree.

On the other hand, each color $i \in\{1, \ldots,(\Delta+1)\}$ is used on the edges of $H_{\Delta+1}$ exactly $\frac{\Delta-2}{2}$ times, i.e., it is not used on the edges incident on the vertex with the color $i$ and it is not used at any other vertex. We name $g(i)$ the color of this vertex, then a color $i \in\{1,2, \ldots,(\Delta+1)\}$ appears at the edges of $G$ exactly

$$
k=\frac{|V|-\left(\left|V_{i}\right|+\left|V_{g(i)}\right|\right)}{2}
$$

times, but $\left|V_{i}\right|=\left|V_{g(i)}\right|$, then $k=\frac{|V|-\left(2\left|V_{i}\right|\right)}{2}$, and as $\left|V_{i}\right|=\frac{|V|}{\Delta+1}$ it follows that

$$
|V|-2\left|V_{i}\right|=|V|-2 \frac{|V|}{\Delta+1}=\frac{(\Delta-1)|V|}{\Delta+1}, \text { where } k=\frac{(\Delta-1)|V|}{2(\Delta+1)} .
$$

Now, we have that color $i$ is used at the total coloring

$$
k=\frac{|V|}{\Delta+1}+\frac{(\Delta-1)|V|}{2(\Delta+1)}=\frac{1}{\Delta+1}\left(|V|+\frac{(\Delta-1)|V|}{2}\right)=\frac{1}{\Delta+1} \frac{(\Delta+1)|V|}{2}=\frac{|V|}{2}
$$

times, so the coloring is equitable.

Now, we show how to construct some Harmonic Graphs which are Cartesian product of Cycles and their complements.

Theorem 5. The Cartesian product of a Cycle Cn and its complement is an Harmonic Graph.

Proof. First, we note that $K_{m+1}$ satisfies the conditions of the theorem.

For $n=3$, the theorem is trivial, because the vertex coloring obtained from the cycle $C_{3}$ satisfies the conditions of the theorem, i.e. the $C_{3}$ naturally admits a range coloring of order 2 , with three colors $c_{1}, c_{2}$ and $c_{3}$. 
Let be an arbitrary natural number $m$. Consider a cycle $C_{n}^{1}, n=m+1$. Let be $V=\left\{v_{1}, \ldots, v_{n}\right\}$ its vertices and $C=\left\{c_{1}, \ldots, c_{n}\right\}$ a set of colors. Without loss of generality, we assume that the $n$ vertices of the cycle were colored by the mapping $f_{1}: V\left(C_{n}^{1}\right) \rightarrow C$, where, $f_{1}\left(v_{i}\right)=c_{i}$, $i=1, \ldots, n$.

The next step is to construct $(n-1)$ copies $C_{n}^{2}, K, C_{n}^{m}$ of $C_{n}^{1}$, and define the coloring $f_{i}$ : $V\left(C_{n}^{i}\right) \rightarrow C$, where $f_{i}\left(v_{j}\right)=c_{k}, k=(j+(n-i)) \bmod n+1, i, j=1, \ldots, n$.

Note that for each vertex $v_{j}$ in the cycle $C_{n}^{i}$, the colors used on their neighbors in the cycle are the same ones used to color their copies in cycles $C_{n}^{(i+1)} \bmod n$ and $C_{n}^{(i-1)} \bmod n$. The neighbors are exactly the vertices adjacent to vertex $v_{j}$ in $C_{n}^{i}$. For each remaining $(n-3)$ copies of $v_{j}$, different colors were applied, which are different of the ones that were used in $v_{j}$ and its neighbors in the cycle $C_{n}^{i}$. We can add an edge joining $v_{j}$ to each of its copies, which are located at different cycles of $C_{n}^{i}, C_{n}^{(i+1) \bmod n}$, and $C_{n}^{(i-1)} \bmod n$. The resulting coloring is also a range color of $\operatorname{order} \Delta$.

Originally, the graph was 2-regular and $(n-3)$ edges were added at each vertex. The resulting graph is regular of degree $(n-1)=m$, colored with $n=(\Delta+1)$ colors and range $(n-1)$. Then it is an Harmonic Graph.

From Theorem 4, we have that the total coloring of the Cartesian Product of a Cycle $C_{n}$ and its complement is equitable. Figure 5, bellow, illustrates the equitable total coloring of Cycle $C_{n}$ and its complement.

Observe that it is always possible to increase the degree of this kind of Harmonic Graph while keeping the equitable total coloring. Let $G(V, E)$ be an $n$-harmonic graph, constructed from a cycle, and $c: V \rightarrow C$ an equitable coloring of $G$ using $\Delta(G)+1$ colors. We will construct a graph $G^{\prime}\left(V^{\prime}, E^{\prime}\right)$ as follows:

Let be $V^{\prime}=V \cup\left\{u_{11}, \ldots, u_{1 n}, \ldots, u_{j 1}, \ldots, u_{j n}, \ldots, u_{s 1}, \ldots, u_{s n}\right\}$ and $E_{i}=\{e=\{x, y$ : $x=u_{j i}$ and $\left(y=u_{k i}\right.$ or $\left.\left.\left.y=v_{t i}\right) ; t, k \in\{1, \ldots, n\}, j \in\{1, \ldots, s\}, k \in j\right\}\right\}$, where $v_{1 i}, \ldots, v_{n i}$ are the vertices of the cycle $C_{n}^{i}, i=1, \ldots, n$. Let $E *=\bigcup_{i=1}^{n} E_{i}$ and $E^{\prime}=E \cup E * . G^{\prime}$ satisfies the conditions of Theorem 5. In fact, $\Delta\left(G^{\prime}\right)=\Delta(G)+m$, then $c^{\prime}(v)= \begin{cases}c(v) & \text { if } v \in V \\ c_{\Delta+j+1} & \text { if } v=u_{j i}\end{cases}$ is an equitable coloring of $G^{\prime}$ using $\Delta\left(G^{\prime}\right)+1$ colors.

Figure 6, bellow, shows the equitable total coloring of a 5-regular graph from a harmonic graph, constructed from a 5-cycle.

\section{CONCLUSIONS}

The equitable coloring is valuable because it can be used in task allocation in general, in order to guarantee the balance in the distribution of tasks. Thus, the Harmonic Graphs have relevant characteristics for admitting a total equitable coloring that meets the Vizing-Behzad Conjecture, as demonstrated in Theorem 2. As these graphs necessarily require a range coloring of order $\Delta$, 


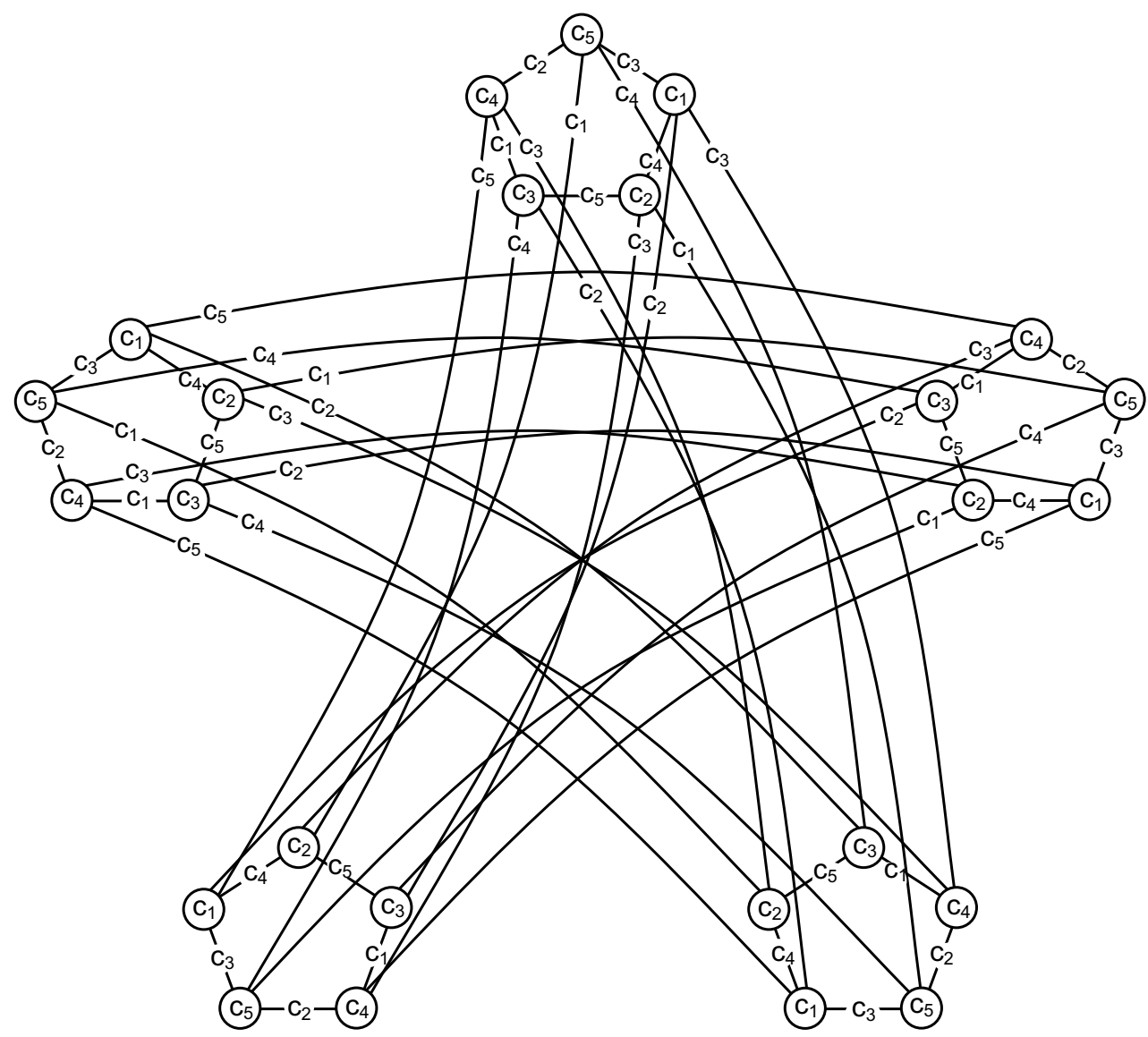

Figure 5 - Equitable total coloring of the Cartesian product of C5 and its complement.

it is likely to emerge the following questions: Besides the Complete Graphs, are there any other Harmoni Graphs? Can we build Harmonic Graphs, easily?

The Cycles whose number of vertices is a multiple of three are examples of Harmonic Graphs, as well as the Cube. In this paper, we have presented an efficient way to get other Harmonic Graphs: we constructed Harmonic Graphs which are Cartesian products of Cycles and their complements. Such graphs, in general, show an interesting relation between a graph of few edges (Cycle) with other of many edges (the complement of the Cycle). The generation of Harmonic Graphs is not limited only to the ones shown along this paper. In case the reader is interested to obtain further information, consult Lozano et al. (2013), as already mentioned.

In the upcoming works, we intend to generalize the results we have presented within this paper in order to build Harmonic Graphs that are more complex than the Cartesian product of Cycles and their complements. We are also willing to study the possibility of recognizing subfamilies of Harmonic Graphs. 


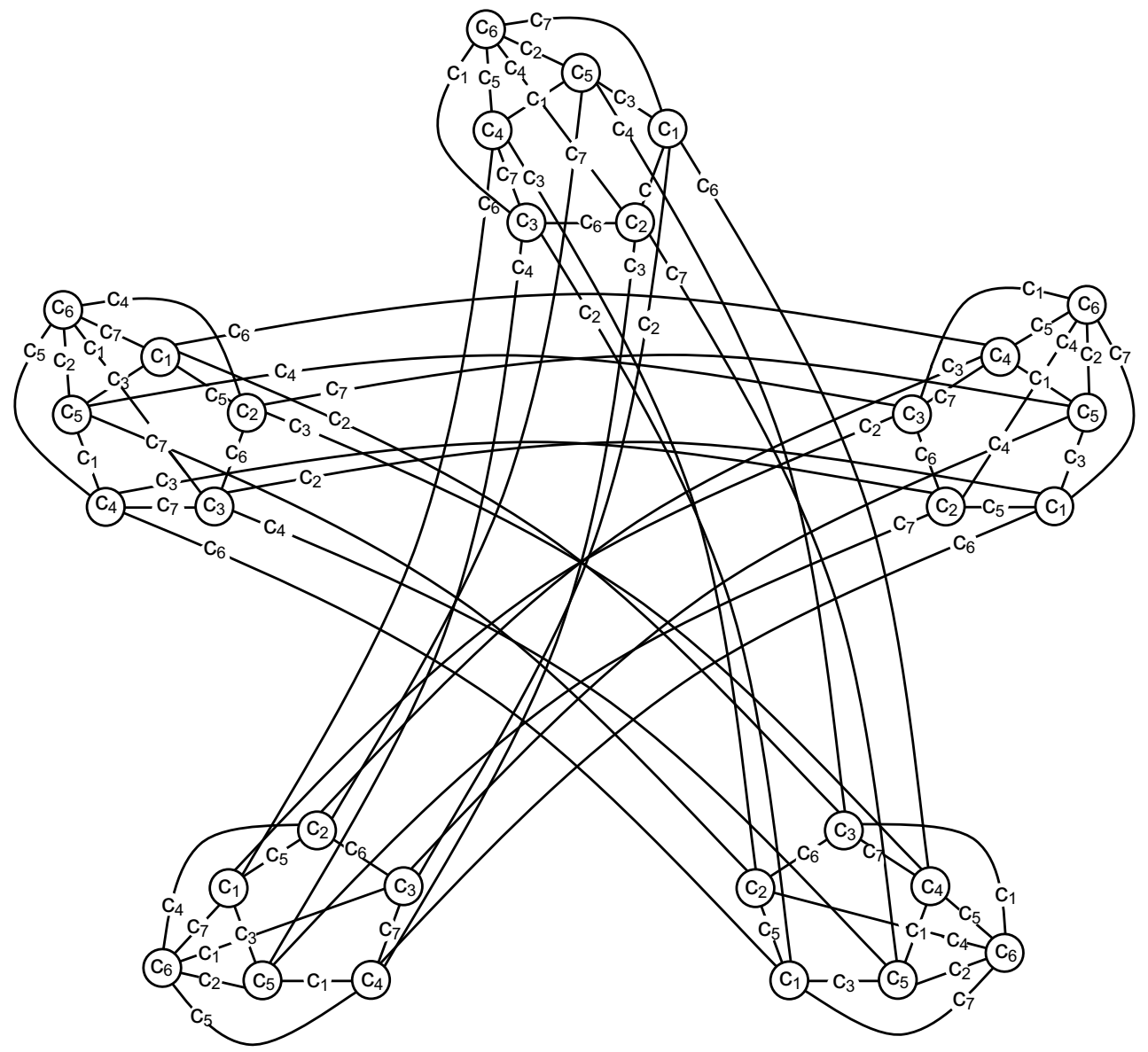

Figure 6 - Equitable total coloring of a 5-regular graph from a 5-harmonic.

\section{REFERENCES}

[1] Bondy JA \& MURTY USR. 1976. Graph Theory with Applications. New York: North-Holland.

[2] ChEN BL \& LiH KW. 1994a. Equitable coloring of trees. Journal of Combinatorial Theory, Series B, 61: 83-87.

[3] Chen BL, Ko MT, Lih KW \& Wu PL. 1994b. Equitable coloring and the maximum degree. European. J. Combinatorics, 15: 443-447.

[4] Chen BL, Ko MT, Lin KW \& WU PL. 1996. Equitable and m-bounded coloring of split graphs. Combinatorics and Cumputer Science, Lecture Notes in Computer Science, 1120: 1-5.

[5] Chunling T, Xiaohui L, Yuansheng Y \& Zhine L. 2009. Equitable Total Coloring de $C_{m} \square C_{n}$. Discrete Applied Mathematics, 157: 596-601.

[6] Diestel R. 1997. Graph theory. New York: Springer-Verlag. 
[7] Friedmann CVP, Markenzon L, Lozano ARG \& Waga C. 2011. Total Coloring of BlockCactus Graph. Journal of Combinatorial Mathematics and Combinatorial Computing, 78: 273-283.

[8] Hajnal A \& Szemerédi E. 1970. Proof of a conjecture of Erdös. Combinatorial Theory and Its Applications 2. In: Colloq. Math. Soc. János Bolyai 4, Amsterdam, Netherlands: North-Holland, 601-623.

[9] Hilton AJW \& WerRa D. 1994. A sufficient condition for equitable edgecolourings of simple graphs. Discrete Math., 128: 179-201.

[10] Kemnitz A \& Marangio M. 2003. Total colorings of cartesian products of graphs. Congres. Numer., 165: 99-109.

[11] LoZANo ARG. 2005. Coloração total equilibrada de grafos. Tese de Doutorado, COPPE/UFRJ, Rio de Janeiro, RJ, Brasil.

[12] Lozano ARG, Friedmann CVP \& Jurkiewicz S. 2008. Coloração Total Equilibrada - Um Modelo para Redes de Interconexão. Pesquisa Operacional, 28(1): 161-171.

[13] Lozano ARG, Friedmann CVP, Waga C \& MARKenzon L. 2009. Coloração de Vértices com Folga. In: XLI Simpósio Brasileiro de Pesquisa Operacional, Porto Seguro. Anais do XLI SBPO, 3084-3091.

[14] Lozano ARG, Siqueira AS, Jurkiewicz S \& Friedmann CVP. 2013. Produto Funcional de Grafos. Tendências em Matemática Aplicada e Computacional, 14(2): 1-12.

[15] Seoud MA, Abd El Maqsoud Aei, Wilson RJ \& Williams J. 1997. Total colouring of cartesian products. Int. J. Math. Educ. Sci. Technol., 4(28): 481-487.

[16] SiqueIRA AS. 2011. Coloração total equilibrada em subfamílias de grafos regulares. Tese de Doutorado, COPPE/UFRJ, Rio de Janeiro, RJ, Brasil.

[17] WANG WF \& ZHANG K. 2000. Equitable colorings of line graphs and complete r-partite graphs. System Sci. Math. Sci., 13: 190-194.

[18] WANG WF. 2002. Equitable Total Colorings of graphs with Maximum Degree 3. Graph and Combinatorics, 18: 677-685.

[19] YAP HP. 1986. Some topics in graph theory. London: Cambridge University Press.

[20] YAP HP. 1996. Total colorings of graphs. Berlin: Springer.

[21] ZMAZEK B \& ZerovniK J. 2002. Behzad-Vizing conjecture and Cartesian product graphs. J. Appl. Math. Lett., 15: 781-784. 